\title{
Continuous High-Frequency Stimulation of the Subthalamic Nucleus Improves Cell Survival and Functional Recovery Following Dopaminergic Cell Transplantation in Rodents
}

Neurorehabilitation and Neural Repair 2015, Vol. 29(10) 1001-1012 (C) The Author(s) 2015 Reprints and permissions: sagepub.com/journalsPermissions.nav DOI: I0.1 I77/I5459683/558|419 nnr.sagepub.com @SAGE

\author{
Luciano L. Furlanetti, MD', Joacir Graciolli Cordeiro, MD, PhD', \\ Karina Kohn Cordeiro, MD, PhD', Joanna A. García, PhD ${ }^{1,2}$, \\ Christian Winkler, MD, PhD ${ }^{1,3}$, Guilherme A. Lepski, MD, PhD ${ }^{4,5}$ \\ Volker A. Coenen, MD', Guido Nikkhah, MD, PhD', and Máté D. Döbrössy, PhD'
}

\begin{abstract}
Subthalamic nucleus (STN) high-frequency stimulation (HFS) is a routine treatment in Parkinson's disease (PD), with confirmed long-term benefits. An alternative, but still experimental, treatment is cell replacement and restorative therapy based on transplanted dopaminergic neurons. The current experiment evaluated the potential synergy between neuromodulation and grafting by studying the effect of continuous STN-HFS on the survival, integration, and functional efficacy of ventral mesencephalic dopaminergic precursors transplanted into a unilateral 6-hydroxydopamine medial forebrain bundle lesioned rodent PD model. One group received continuous HFS of the ipsilateral STN starting a week prior to intrastriatal dopaminergic neuron transplantation, whereas the sham-stimulated group did not receive STN-HFS but only dopaminergic grafts. A control group was neither lesioned nor transplanted. Over the following 7 weeks, the animals were probed on a series of behavioral tasks to evaluate possible graft and/or stimulation-induced functional effects. Behavioral and histological data suggest that STN-HFS significantly increased graft cell survival, graft-host integration, and functional recovery. These findings might open an unexplored road toward combining neuromodulative and neuroregenerative strategies to treat severe neurologic conditions.
\end{abstract}

\section{Keywords}

6-hydroxydopamine, Parkinson's disease, cell therapy, deep brain stimulation, subthalamic nucleus

\section{Introduction}

Chronic high-frequency stimulation (HFS) of the subthalamic nucleus (STN) has been validated for use in the treatment of Parkinson's disease (PD). ${ }^{1,2}$ The efficacy of STN-HFS in relieving the cardinal motor symptoms of PD is long-lasting and is considered by some to be superior to the best medical therapy available..$^{2-5}$ The mechanisms by which electrical stimulation of a deep brain structure reinstates/modulates motor function are not completely unknown, and it is not believed to involve lesioning, given that interrupting stimulation can reverse its effects. ${ }^{6,7}$ Improving on current preclinical models has facilitated our understanding of the underlying mechanisms, particularly with respect to assessing the capacity of HFS to modulate motor and sensorimotor functions.
Cell replacement therapy strategies using embryonic ventral mesencephalic (VM) dopaminergic cells grafted into the striatum have not been clinically shown to be as effective as deep brain stimulation (DBS) in ameliorating

\footnotetext{
'University Freiburg Medical Center, Freiburg im Breisgau, Germany ${ }^{2}$ Columbia University, New York, NY, USA

${ }^{3}$ Lindenbrunn Hospital, Coppenbrügge, Germany

${ }^{4}$ University of São Paulo, São Paulo, Brazil

${ }^{5}$ University of Tübingen, Tübingen, Germany

${ }^{6}$ University Hospital of Erlangen, Erlangen, Germany

\section{Corresponding Author:}

Luciano L. Furlanetti, Laboratory of Stereotaxy and Interventional Neurosciences, Department of Stereotactic and Functional

Neurosurgery, University Freiburg Medical Center, Breisacher Str 64, Freiburg 79106, Germany.

Email: luciano.furlanetti@uniklinik-freiburg.de
} 


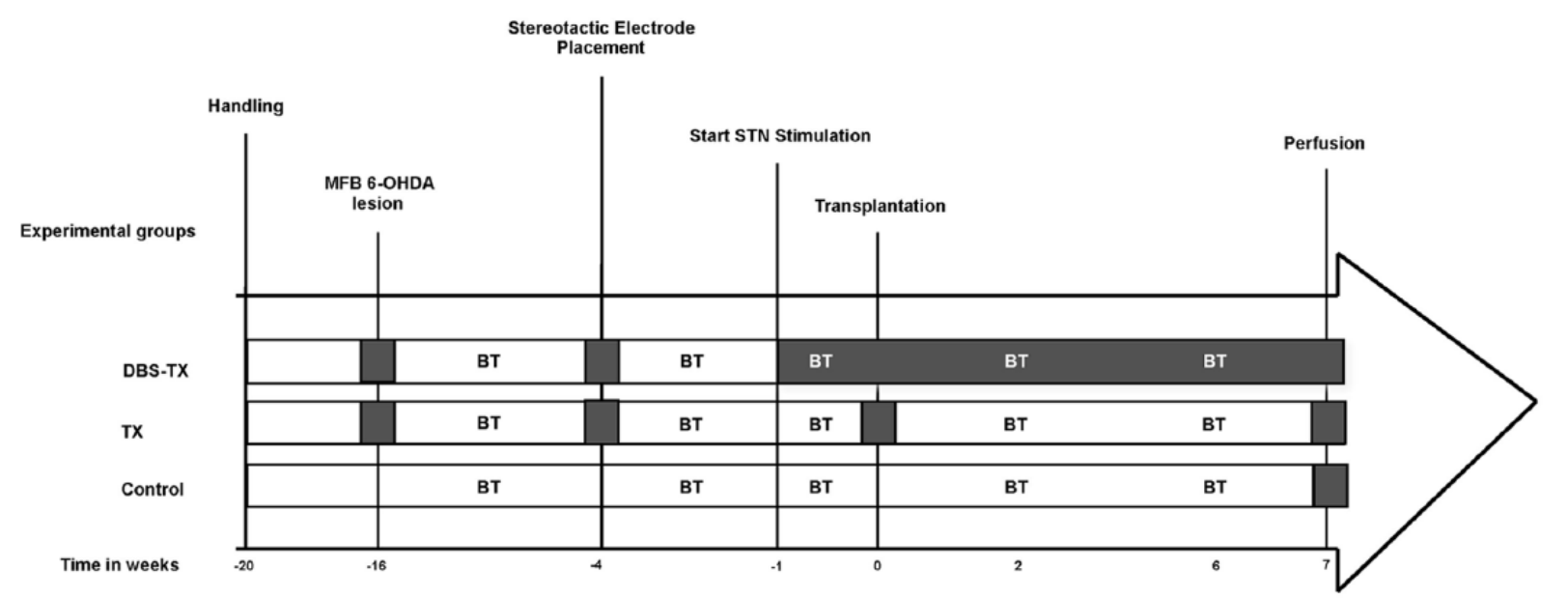

Figure I. Experimental design and timeline of an in vivo experimental model of Parkinson's disease.

6-OHDA, 6-hydroxydopamine; BT, behavior test session (ie, drug-induced rotation, cylinder test, stepping test); MFB, medial forebrain bundle; STN, subthalamic nucleus.

L-DOPA-responsive PD motor symptoms. However, this approach remains a potential treatment for PD, as it is based on partially restoring the lost dopaminergic neurons and some of their functions. ${ }^{8-10}$ Clinical trials evaluating the role of cell therapy in the treatment of PD have shown that the success of VM grafts depends on aspects of the transplantation procedure (eg, patient selection, tissue dissection, handling, and storage), postoperative immunosuppressive treatments, and the number of surviving dopaminergic cells in the graft. Thus, a central issue for the success of cell therapy-based approaches seems to be the maintenance of optimal conditions for graft survival. ${ }^{8,10}$ A recent report shows that lesion of the STN led to a significant increase in the number of surviving dopamine grafted cells in the host striatum and improved functional outcome, though this improvement was limited to a drug-induced rotation task. ${ }^{11}$ It is not known whether chronic electric stimulation of the STN is able to reproduce or even boost this effect, although electrical inputs to a neurogenic circuit, for example, the hippocampal perforant pathway, can enhance local neurogenesis, accelerate maturation of granule cells, and foster neosynaptogenesis. ${ }^{12,13}$ Thus, further investigation is needed to determine whether chronic electrical stimulation induces neurogenesis in exogenous cell grafts. Studies involving STN lesion and STN-HFS following nigrostriatal 6-OHDA-induced lesions revealed increased nigral dopamine expression and reduced neuronal loss compared to controls, documenting a neuroprotective effect of STN modulation in this model. ${ }^{14,15}$ Nevertheless, the electrophysiological and behavioral aspects of this technique have yet to be explored.

The current study assessed whether STN neuromodulation and cell therapy exert a synergistic positive effect on graft survival and functional recovery in a rodent model of PD. Our results suggest that STN-HFS can be neuroprotective, influencing positively both the survival of the grafted cells as well as the animals' behavioral performance.

\section{Methods}

\section{Study Design}

Female Sprague Dawley rats weighing between 250 and 350 grams ( $\mathrm{n}=30$, Charles River, Germany) were housed in transparent round Plexiglas cages (height $40 \mathrm{~cm}$, diameter $40 \mathrm{~cm}$ ) under a 12-hour light/dark cycle, with food and water ad libitum. After 2 weeks of handling, 20 rats received a unilateral stereotactic injection of 6-OHDA in the right medial forebrain bundle (MFB), leading to complete dopamine depletion in the ipsilateral striatum; the remaining rats (control group, $\mathrm{n}=10$ ) were neither lesioned nor received any treatment and served as behavioral and histological controls. Following MFB lesion, animals were matched and groups balanced according to their performance in the Amphetamineinduced rotation and Cylinder Tests. Next, all lesioned animals were implanted with microelectrodes, and 2 weeks following a recovery period, STN stimulation started only in the DBS-TX group. A week after continuous STN-HFS, both the DBS-TX ( $\mathrm{n}=8$; lesioned, grafted, stimulated) and the TX $(n=10$; lesioned, grafted, unstimulated) groups received intrastriatal fetal ventral mesencephalic grafts. Experimental procedures are summarized in Figure 1.

The experiment was approved by the local ethics committee and followed the ethical guidelines set by the Regierungspraesidium Freiburg (Tierversuchsantrag G10/124). 


\section{6-OHDA MFB Lesion}

Animals were anaesthetized using gaseous isoflurane (4\% during induction and $1.8 \%$ to $2.5 \%$ for maintenance), placed in a stereotactic frame (Stoelting, Germany) and injected with $6-\mathrm{OHDA}(3.6 \mu \mathrm{g} / \mu \mathrm{L}$ in $0.2 \%$ ascorbic acid and $0.9 \%$ saline; Sigma, St Louis, MO) unilaterally in 2 injection tracks in the MFB at the following coordinates relative to bregma: track 1, tooth bar $(\mathrm{TB})=+3.4$; anteroposterior $(\mathrm{AP})=-4.0$; lateral $(\mathrm{L})=-0.8$; dorsoventral $(\mathrm{DV})=-8.0$; and track 2 , TB $=-2.3 ; \mathrm{AP}=-4.4 ; \mathrm{L}=-0.8 ; \mathrm{DV}=-7.8$. In total, each animal received $5.5 \mu \mathrm{L}$ of solution at a rate of $1 \mu \mathrm{L} / \mathrm{min}$. We used buprenorphin $(0.05 \mathrm{mg} / \mathrm{kg} \mathrm{sc})$ for postoperative analgesia. ${ }^{11}$

\section{Electrode Implantation}

Teflon-coated platinum/iridium bipolar electrodes (diameter of $125 \mu \mathrm{m}, 500 \mu \mathrm{m}$ distance between the tips; World Precision Instruments, Sarasota, FL) were implanted in the right STN of the rats in the DBS-TX group at the following coordinates: $\mathrm{TB}=-3.3 ; \mathrm{AP}=-3.6 ; \mathrm{L}=-2.4 ; \mathrm{DV}=-8.5$, with respect to the bregma. The electrodes were connected to a multichannel plug and the implant was fixed to the skull with microscrews and dental cement. ${ }^{16}$ In order to preserve the same conditions for the testing groups, the TX group was also submitted to this procedure.

\section{Transplantation of EI 4 VM Cells}

Embryonic day E14 (crown-rump length $=10.0-11.0 \mathrm{~mm}$ ) rat tissue was used to prepare a single-cell suspension of ventral mesencephalon. Embryos were removed from anesthetized pregnant rats, the VMs were microscopically dissected as previously described, ${ }^{17}$ and a single-cell suspension was prepared according to a modified protocol. ${ }^{18}$ The resulting single-cell suspension was adjusted to a concentration of 130000 cells $/ \mu \mathrm{L}$ in Dulbecco's modified Eagle's medium $/ 0.05 \%$ DNase medium. Cell viability was assessed using the trypan blue dye extraction method. Transplantation was stereotacticaly performed using a $2 \mu \mathrm{L}$ Hamilton syringe fitted to a glass capillary $(\varnothing 50-70 \mu \mathrm{m})$. Two injection tracks with 2 deposits in each were made $(\mathrm{TB}=0.0 ; \mathrm{AP}=+0.2 ; \mathrm{L}$ $=-2.5 /-3.6 ; \mathrm{DV}=-5.0 /-4.0)$. In each deposit, $0.5 \mu \mathrm{L}$ of the single-cell suspension was injected, resulting in 260000 cells per striatum. Rats from each group were operated alternately during the transplantation session in order to control for any deterioration of the cell suspension. Dissection of the $\mathrm{VM}$, preparation of the cell suspension, and stereotactic transplantation were all carried out on the same day.

\section{Deep Brain Stimulation}

Fourteen days after electrode implantation, animals were connected to an external pulse generator (STG 2008, Multichannel Systems, Reutlingen, Germany) for continuous high-frequency stimulation of the right STN (DBS-TX group) or sham stimulation (TX group) for 7 weeks. Stimulus pulses consisted of square-wave biphasic, constant current pulse pairs, and stimulation was started using clinically relevant parameters (frequency $130 \mathrm{~Hz}$, pulse width $60 \mu \mathrm{s}$, and amplitude $175 \mu \mathrm{A}$; Figure 2a and d).

\section{Behavioral Tests}

Drug-Induced Rotation. The drug-induced rotation was performed at 5 time points: (a) 2 weeks post-MFB lesion; (b) 4 weeks post-electrode implantation; $(c) 1$ week post-STNHFS; $(d) 2$ weeks postgrafting; and (e) 6 weeks postgrafting, when the test was carried both with and without HFS. The rats were placed in rotometer bowls connected to a computer. A customized system was adapted from the original design of Ungerstedt, ${ }^{19}$ allowing the test to be carried out under continuous HFS (Figure 2b). Ipsilateral and contralateral rotations were recorded over 90 minutes after amphetamine injection $(2.5 \mathrm{mg} / \mathrm{kg}$ ip, Sigma; in $0.9 \%$ saline). Two days later, the apomorphine-induced rotation was carried out (40 minutes, $0.05 \mathrm{mg} / \mathrm{kg}$ sc injection, Sigma; in $0.2 \%$ ascorbic acid and $0.9 \%$ saline). Data are presented as net ipsilateral or contralateral turns/minute. ${ }^{11}$

Cylinder Test. Forelimb movement asymmetry and exploratory behavior were tested with the cylinder test $\mathrm{s}^{20,21} 6$ weeks after MFB lesion and following every intervention, as described above. The animals were placed in the center of a $20-\mathrm{cm}$ diameter clear Plexiglas cylinder and their exploratory behavior was recorded with a camera for 4 minutes. We counted the number of times the animal made clear contact with the cylinder wall, using either the ipsilateral or the contralateral forepaw, and calculated use of the contralateral paw as a percentage of the total touches with both paws (Figure 2c).

Stepping Test. Forelimb akinesia was tested using the stepping test, ${ }^{22,23}$ which was carried out 6 weeks following the MFB lesion and after each intervention, in the same fashion as for the other tests. In this task, the rat is slowly moved 60 $\mathrm{cm}$ sideways over a flat surface: with one forelimb restrained, we count the number of adjusting steps made by the unrestrained paw. Stepping data are presented as the number of adjusting steps made by the unrestrained paw.

\section{Immunohistochemistry and Histological Analysis}

After 7 weeks of continuous STN stimulation and/or striatal transplantation, the rats were terminally anaesthetized and transcardially perfused, using $300 \mathrm{~mL}$ ice-cold $1 \times$ phosphatebuffered saline (PBS), followed by $300 \mathrm{~mL}$ ice-cold $4 \%$ paraformaldehyde (PFA). Removed brains were post-fixed by immersion in 4\% PFA for 12 hours, transferred to $30 \%$ sucrose solution, and left at $4^{\circ} \mathrm{C}$ until they sunk. Coronal 


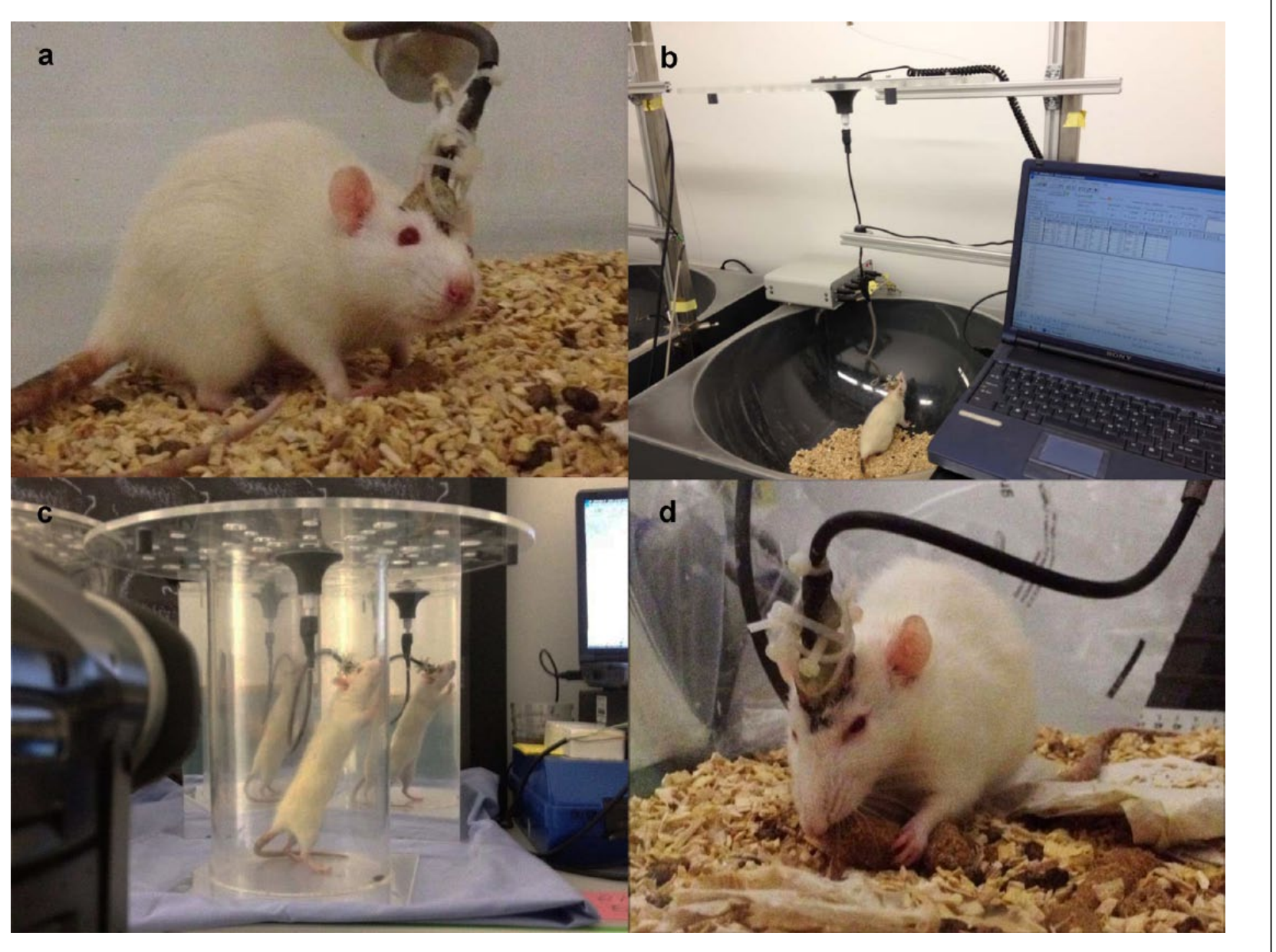

Figure 2. DBS platform in rodents and behavioral tests adapted for chronic continuous high-frequency stimulation (HFS).

(a) and (d) 6-OHDA rat model of PD in the home cage during the experiment. (b) Drug-induced rotation test and (c) Cylinder test setups adapted to be performed under continuous STN-HFS. 6-OHDA, 6-hydroxydopamine; PD, Parkinson's disease; STN, subthalamic nucleus.

sections of $40-\mu \mathrm{m}$ thickness were cut on a microtome and collected in antifreeze solution.

Endogenous peroxidase activity was quenched by incubating the sections for 5 minutes in $3 \% \mathrm{H}_{2} \mathrm{O}_{2}$ and $10 \%$ methanol in PBS. Nonspecific binding was blocked by 1-hour pre-incubation in 5\% normal serum containing $0.25 \%$ Triton X-100 in PBS, followed by incubation with the appropriate primary antibody in the same mixture of serum and Triton X-100 as follows: mouse anti-tyrosine hydroxylase (1:2500; Sigma-Aldrich, Steinheim, Germany), mouse-anti-NeuN (1:250; Merck Millipore MAB377, Darmstadt, Germany), mouse-anti-rat Monocytes/ Machophages (ED-1, 1:1000; Merck Millipore MAB1435, Darmstadt, Germany), or polyclonal rabbit anti-Ki67 (1:1000; Novocastra, Germany). The primary antibody was incubated overnight at room temperature. Then, sections were rinsed 3 times with $1 \times$ PBS, followed by 1 -hour incubation in the appropriate biotinylated secondary antibody (rabbit-anti-mouse IgG biotinalated, diluted 1:200, Dako E0464) and 1-hour incubation with avidin-biotin peroxidase solution (ABC Elite; Vector Laboratories). Finally 3,3-diaminobenzidine (DAB; Merck, Darmstadt, Germany) and $0.01 \% \mathrm{H}_{2} \mathrm{O}_{2}$ were used to develop the color reaction.

The grafted TH-positive cells were counted in the striatum, using a Leica DMRB microscope and the Stereoinvestigator software (MFB Bioscience, Williston, VT). The total number was estimated using Abercrombie's correction formula $\mathrm{P}=\mathrm{M} /(\mathrm{D}+\mathrm{M}) \mathrm{A} * \mathrm{~N}$, where $\mathrm{P}=$ total cell number, $\mathrm{M}=$ section thickness (here, $40 \mu \mathrm{m}$ ), $\mathrm{D}=$ average diameter of the TH-positive cells, $\mathrm{A}=$ number of counted cells, and $\mathrm{N}=$ number of cut series (6). Dopaminergic reinnervation of the depleted striatum was estimated by calculating the optical density (OD) of the grafts using an Olympus Ax-70 microscope and the Analysis software (Soft Imaging Systems, Germany). TH fiber density expressed as a percentage (grafted striatum relative to intact 
striatum) was calculated by assessing the difference in OD between the graft and the corpus callosum (internal control), and the intact striatum and the corpus callosum, and it was calculated on 4 selected AP coordinates $(+1.0 ;+0.2$; $-0.4 ;-1.0)$. The OD of the grafted striatum was expressed as a proportion of the contralateral side: (lesioned striatum OD - corpus callosum OD)/(intact striatum OD - corpus callosum OD) * $100 .^{11,20}$ Graft volume was also calculated. ${ }^{20} \mathrm{ED}-1$ (in the host striatum) and Ki67 immunostainings (within the graft/host striatum, in the subventricular zone [SVZ], in the rostral migratory stream [RMS], and in the dentate gyrus [DG]) were quantitatively assessed by stereological analysis at $40 \times$ magnification. Electrode position was assessed using NeuN staining. STN electrode placement was considered adequate when the gap between the tips of the bipolar electrode was found within the anatomical delimitation of the STN. ${ }^{16}$

\section{Statistical Analysis}

Experimental groups and behavioral testing sessions were used as main factors for 1- and 2-way ANOVAs. The Newman-Keuls test was applied post hoc when a main group effect was detected. Statistica (StatSoft, Inc, 1997) and Sigma Plot (SPSS, Inc, 2002) software were used for multifactorial analyses of variance. Values are presented as means \pm standard error of the mean (SEM). The first outcome measurement presented is the effect of each intervention (electrode implantation, striatal transplantation, and STN stimulation) on behavioral outcome. Further postmortem immunohistochemistry analyses were conducted and results were compared across groups. Statistical analyses concerning behavioral recovery were performed per session within and across groups.

\section{Results}

\section{STN-HFS Boosts TH+ Cell Count, TH Fiber Outgrowth, and Graft Volume}

Injection of 6-OHDA within the nigrostriatal pathway led to a complete depletion of TH-positive cells in the ipsilateral substantia nigra ( $\mathrm{SN})$ and of dopaminergic projections and terminals in the MFB and the striatum. Animals with incomplete unilateral MFB lesion and/or misplaced STN electrode were excluded from the statistical analysis. The dopaminergic grafts were localized mainly in the rostrolateral portion of the striatum and were marked by the presence of TH-positive cells surrounding the core and fiber extension throughout the host-graft interface (Figure 3a-d). In the DBS-TX group, a robust and significant increment in the number of surviving grafted TH-positive cells was observed in comparison to the TX group $(2085.3 \pm 371.8$ vs $1109.2 \pm$
141.8 , respectively; $F_{1,18}=6.8 ; P=.017$; Figure 4a). Graftderived TH-positive fiber outgrowth in the striatum was quantified by means of optical density measurement. TH-positive fiber density was significantly higher in animals that received STN-HFS and transplantation (DBS-TX group $36.5 \pm 3.3 \%$ vs TX group $26.6 \pm 1.7 \%$ ), (Groups, $F_{1,18}=12.8$; $P=.002$; Figure $4 \mathrm{~b})$. The analysis of graft volume across the groups reinforced the positive effect of STN-HFS on graft development (DBS-TX group $1.75 \pm 0.2$ vs TX group $0.73 \pm$ $0.1 \mathrm{~mm}^{3}$ ), (Groups, $F_{1,16}=32.2 ; P<.001$; Figure $4 \mathrm{c}$ ).

\section{STN-HFS Reduces Graft-Derived Inflammation in the Host Striatum and Increases Neurogenesis}

As presented on the histological plate (Figure 3m-o), Ki67 immunostaining revealed a large number of proliferating cells both in constitutive and potential neurogenic zones. As regard the number of dividing cells in these areas, statistical comparison among groups showed a significantly higher increase in neurogenesis in the DBS-TX group within the striatal graft (DBS-TX group $292.1 \pm 34.9$ vs TX group $116.2 \pm 17.3$ ), (Groups, $F_{2,19}=24.3 ; P<.001$ ) and in the RMS (DBS-TX group $412.3 \pm 39.2$ vs TX group $314.9 \pm$ 18.7), (Groups, $F_{2,19}=5.7 ; P=.01$; Figure $4 \mathrm{e}-\mathrm{f}$ ). No significant changes in Ki67 expression were found in either the SVZ (DBS-TX group $337.0 \pm 27.4$ vs TX group $266.8 \pm$ 23.9), $P=.1$, or the DG (DBS-TX group $16.3 \pm 2.2$ vs TX group $16.8 \pm 1.4), P=.5$; Figure $4 \mathrm{~g}-\mathrm{h}$ ). Local inflammatory changes in the host environment were assessed via ED-1 immunolabeling. Stereological analysis showed an overall increase in the number of striatal ED-1-positive cells (activated microglia) in both transplanted groups. However, a milder reaction in the host striatum/graft in the DBS-TX group was observed (DBS-TX group $3815.3 \pm 336.0$, TX group $8848.6 \pm 1015.2$, control group 118.1 \pm 8.0 ), (Groups, $F_{2,19}=17.9 ; P<.001 ;($ Figure $3 \mathrm{i}-1$ and Figure $4 \mathrm{~d}$ ).

\section{Induced-Rotation Test}

Drug-induced rotation was performed at various time points during the experiment. All animals were submitted to an amphetamine-induced rotation test post-MFB lesion, and the net ipsilateral rotational behavior indicated robust dopamine depletion in the ipsilateral striatum (Figure 5a; Groups $\times$ Sessions, $F_{10,120}=14.5, P=4 \times 10^{-9}$ ). An effect was observed 6 weeks after grafting, both in the DBS-TX group $(P=.0001)$ and in the TX group $(P=.0001)$. Nevertheless, at 2 weeks after transplantation, only the DBS-TX group presented statistically significant improvement in comparison to the pregrafting state (DBS-TX, $P=.0001$; TX group, $P=.058)$. Apomorphine-induced contralateral rotation bias reflected a reduction of postsynaptic receptor sensitivity 

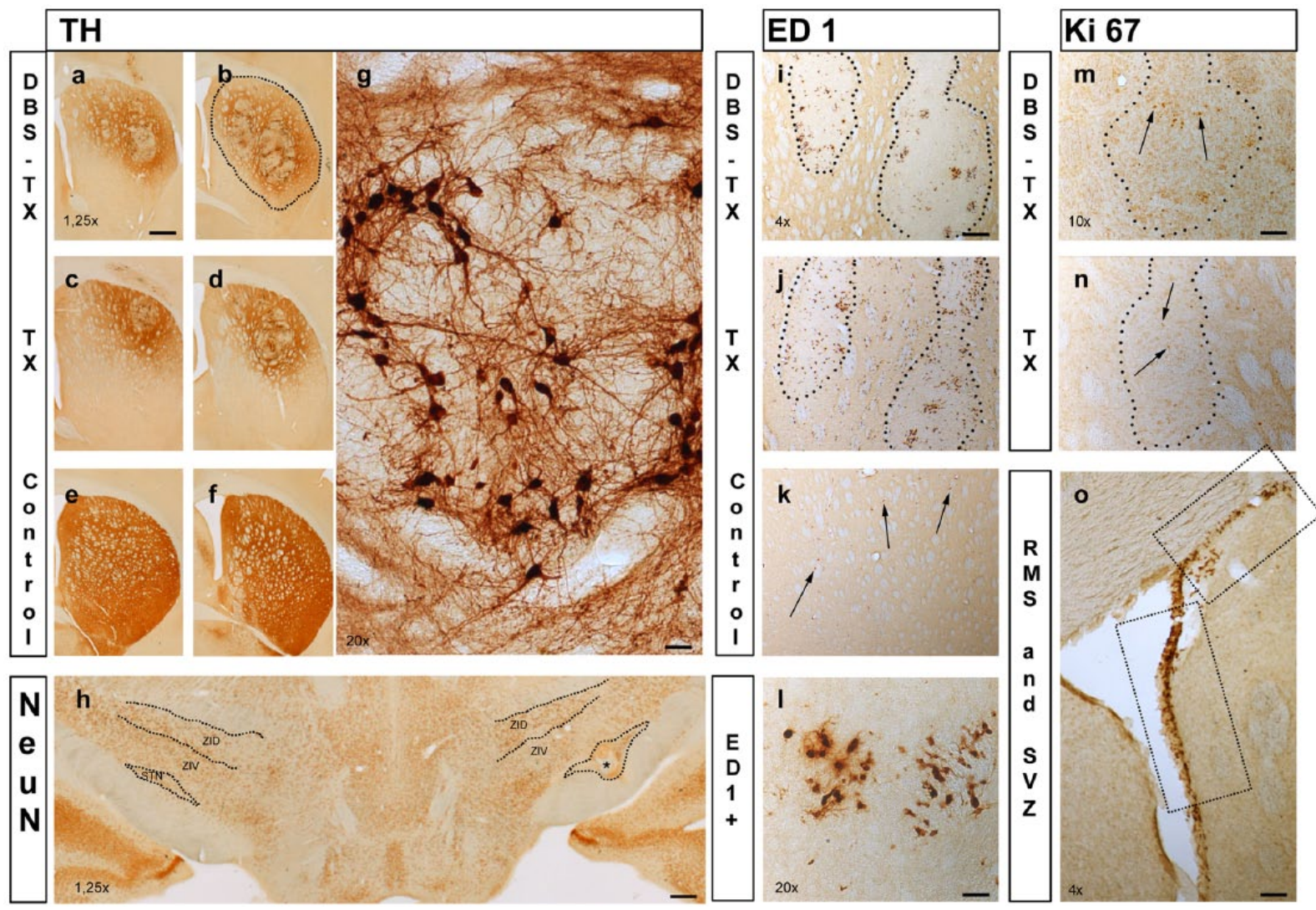

Figure 3. Immunohistochemistry plate.

Upper left: TH staining of coronal sections through the striatum of rodents, showing a substantial positive effect of STN modulation on graft development (I.25×). (a and b) DBS-TX group (dashed line on "b," surrounds the striatal graft); (c and d) TX group; (e and f) Control group. (g) Higher magnification micrograph of TH+ grafted cells, illustrating synaptic connections within the host striatum (20x). Lower left: (h) NeuN staining (coronal section, AP $-3.6 \mathrm{~mm}$ ), for confirmation of the electrode placement into the right STN (*). Right: (i-k) ED-I immunostaining showing increased microglial activation in the grafted host striatum. Graft-induced inflammation was less prominent in the DBS-TX than in the TX group. (I) Microglia (20x); ( $m$ and $n$ ) Ki67 immunolabeling, showing higher cell proliferation index in the DBS-TX group than in the TX group. (o) Ki67 staining revealing constitutive cell proliferation (neurogenesis) in the SVZ and in the RMS. The marked squares indicate the area used for stereological quantification of $\mathrm{Ki} 67+$ cells across the groups. ZID, zona incerta dorsal; ZIV, zona incerta ventral; STN, subthalamic nucleus; SVZ, subventricular zone; RMS, rostral migratory stream.

following transplantation in all grafted groups (DBS-TX, $P$ $=.0001 ;$ TX group,$P=.0001),\left(\right.$ Groups $\times$ Sessions, $F_{2,24}=$ $10.1, P=.0006)$. However, due to ceiling effects, no statistical significance between groups was observed.

\section{Cylinder Test}

Limb-use asymmetry was assessed and translated into functional capacity by analyzing exploratory behavior of the studied groups in a cylindrical chamber at several time points. Restoration of the normal rearing activity and reversing of the limb-use asymmetry caused by dopamine depletion was obtained in both grafted groups (Figure $5 \mathrm{~b}$; Groups $\times$ Sessions, $F_{896}=3.6, P=.0008$ ). Relative to the transplantation-only group, the DBS-TX group obtained significantly greater improvement in contralateral forelimb use at 2 and 6 weeks after transplantation $(P=.02$ and .009 , respectively).

\section{Stepping Test}

The assessment of forelimb akinesia was also performed as a baseline test right after MFB lesion and following every intervention, that is, surgeries, STN-HFS, and/or transplantation. A graft effect was observed at 2 and 6 weeks posttransplantation in both the DBS-TX and TX groups (Forehand: Groups $\times$ Sessions, $F_{896}=9.1, P=2 \times 10^{-9}$; and Backhand: Groups $\times$ Sessions, $F_{8.96}^{8,96}=3.6, P<.001$; Figure $5 \mathrm{c}$ ). A reduced number of adjusting steps of the contralateral forelimb was observed after the 6-OHDA lesion. Both grafted groups improved at 2 and 6 weeks after transplantation. However, the association of STNHFS with striatal transplantation led to a notably faster and 
a.

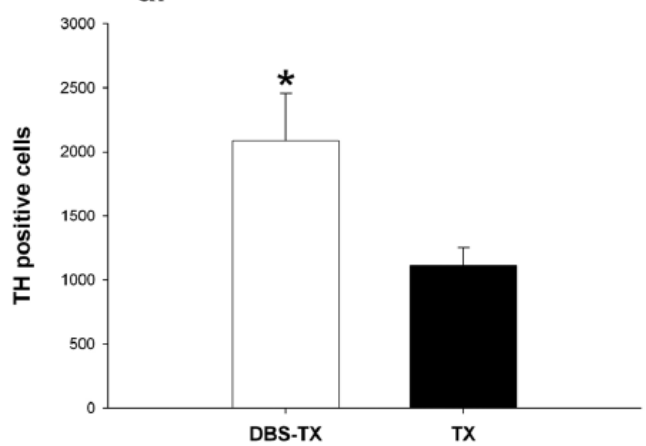

c.

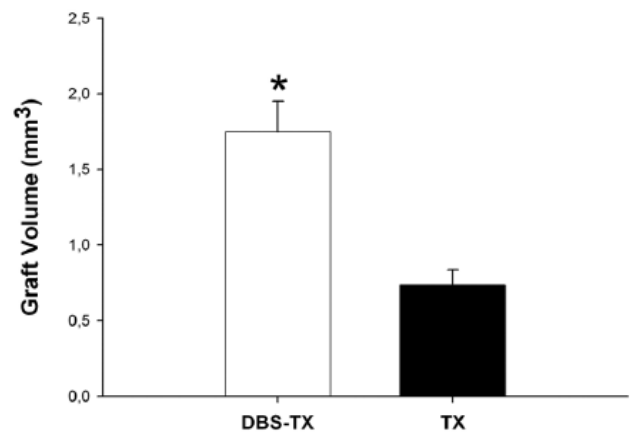

e.

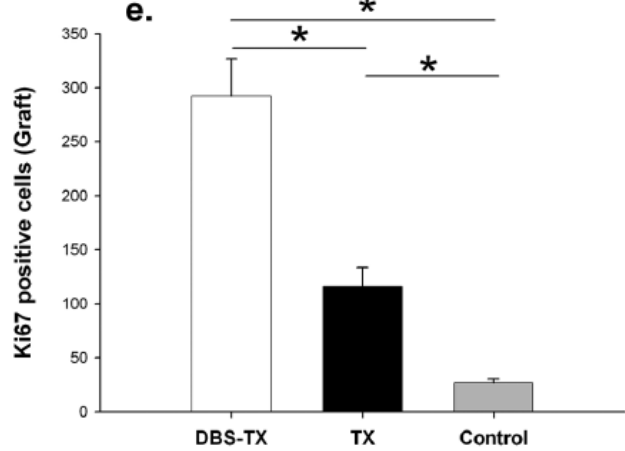

g.

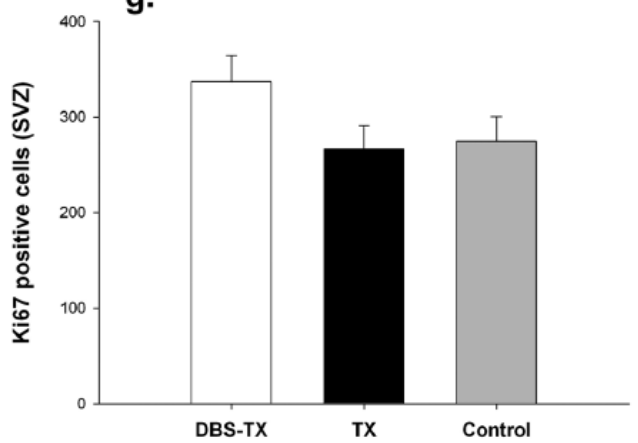

b.

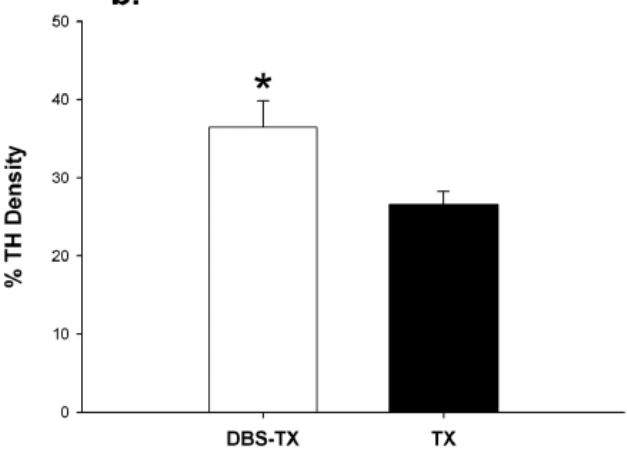

d.
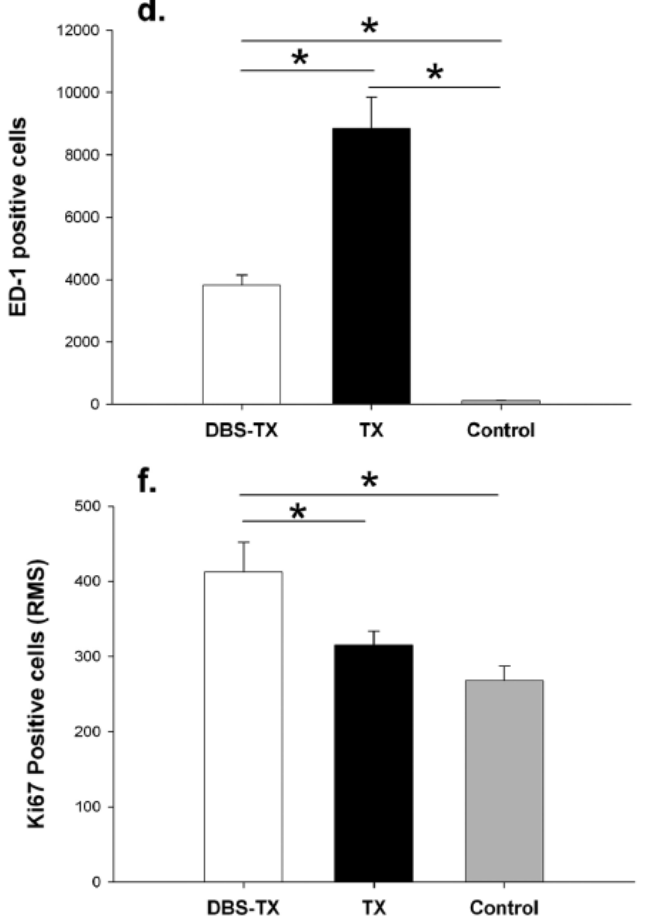

h.

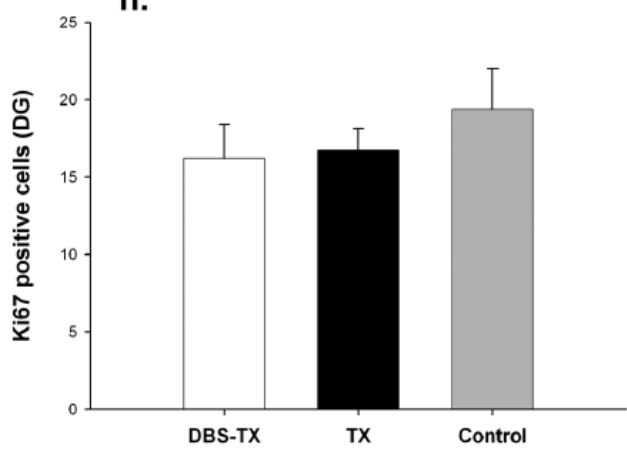

Figure 4. Quantitative assessment of the histological immunostainings.

(a) $\mathrm{TH}+$ cell count. Stereological analysis of tyrosine-hydroxylase $(\mathrm{TH})$-positive cells within the grafted striatum shows a significant increment in the number of grafted TH-positive cells in the DBS-TX group compared to the TX group $(P=.017)$. (b) Fiber density quantification of graft-derived THpositive fiber outgrowth in the striatum. TH-positive fiber density was significantly increased in animals that received STN-HFS + transplant $(P=.002)$. (c) Graft volume evaluation also reinforced a positive effect of STN-HFS on graft development $(P<.00 I)$. (d) ED-I+ cell count revealed an increased number of activated microglia in the grafted striatum, nevertheless with milder inflammatory reaction in the DBS-TX group $(P<.00 \mathrm{I})$. (e-h) Ki67+ cell stereological analysis looked at cell proliferation within the graft and also in constitutional neurogenic areas, such as the RMS, the SVZ, and the DG. A significantly higher increase in neurogenesis in the DBS-TX group within the striatal graft and in the RMS was observed $(P<.00 \mathrm{I}$ and $P=.0 \mathrm{I}$, respectively). No significant changes in Ki67 expression were found in either the SVZ or the DG. Asterisks (*) indicate statistical significance between groups. 

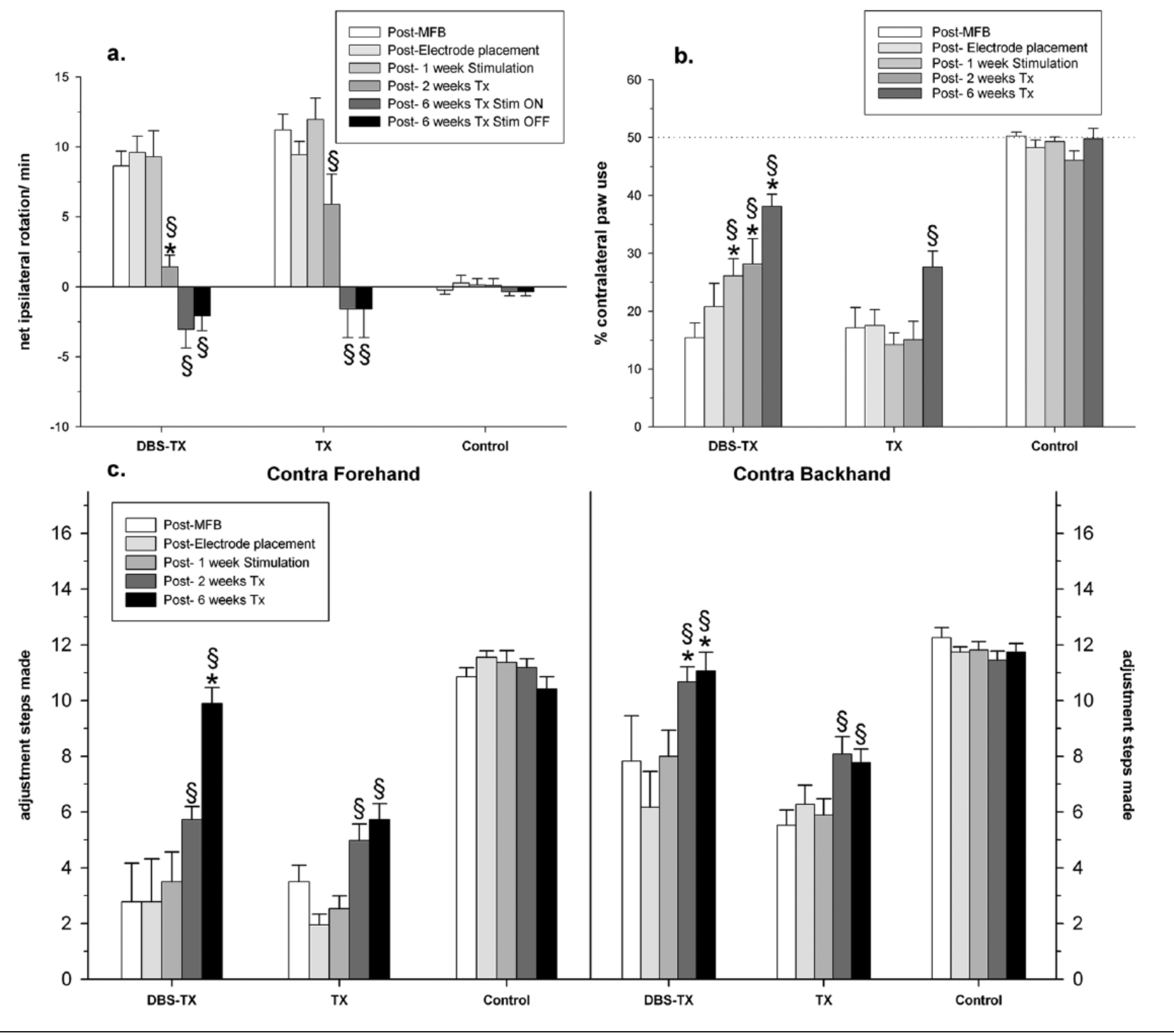

Figure 5. Behavioral assessment performed in several time points along the experiment.

(a) Amphetamine-induced rotation test. A graft effect was observed both in the DBS-TX and in the TX groups, 2 and 6 weeks after transplantation. Two weeks after striatal grafting, the DBS-TX group showed better outcome than the TX group $(P=.047)$. (b) Cylinder test for evaluation of forelimb movement asymmetry and exploratory behavior. Functional improvement on the cylinder test was obtained in both grafted groups. However, the DBS-TX group was significantly better 2 and 6 weeks after transplantation $(P=.009$ and .02 , respectively). "Post-I week stimulation" the DBS-TX group showed a statistically significant better performance in this task than their only-transplanted (TX groups) counterparts $(P=.01)$. (c) Stepping test assessed forelimb akinesia. A reduced number of adjusting steps was observed after 6-OHDA lesion. Both grafted groups improved 2 and 6 weeks after transplantation. The DBS-TX group was significantly better in the contra-forehand task after 6 weeks, and in the contra-backhand test, following 2 and 6 weeks of the transplantation $(P<.01)$. Asterisks $(*)$ indicate statistical significance in the comparison of the same session between groups. $\$$ indicates a "graft effect" within a group, which is always compared with the baseline test (post-MFB lesion).

more robust functional recovery, which was comparable to the performance of the naïve control group, both in the contralateral forehand task after 6 weeks $(P=.001)$ and in the contralateral backhand test after 2 and 6 weeks $(P=.003)$.

\section{Discussion}

Deep brain stimulation in animal models is a well-established and powerful tool for investigating the mechanisms underlying basal ganglia disorders. More recently, DBS has been used to better understand the effects of neuromodulation in the management of several neuropsychiatric diseases. ${ }^{24,25}$ However, the current work is the first experimental attempt to study the effects of chronic STN-HFS combined with cell therapy in a freely moving rat model of PD. At the cellular level, results showed a positive synergistic effect of STN neuromodulation on survival, cell proliferation, and integration of VM dopaminergic grafted cells within the 
host striatum. Moreover, at the behavioral level, striatal grafting together with STN-HFS promoted a vigorous and consistent functional improvement, not only in druginduced rotation tests but also in forelimb use and locomotor activity tasks.

\section{Neuronal Circuitry and Neuromodulation in PD}

Dopaminergic denervation leads to dysfunction of the basal ganglia-thalamocortical circuitry, affecting the dorsolateral and ventromedial loops, and thus disrupting the sensorimotor, associative, and/or limbic pathways; degree of disruption is proportional to the location and extension of the depletion. ${ }^{26}$ Beta-oscillatory synchronization of cerebral cortex, STN, and striatum due to dopamine depletion is now an accepted physiological hallmark of PD. ${ }^{27}$ It has been shown that an increased firing rate in the lower beta-band range $(13-20 \mathrm{~Hz})$ detected by local field potential (LFP) recordings is linked to clinical manifestations of $\mathrm{PD}$, such as bradykinesia and rigidity, and is markedly suppressed by dopaminergic agents ${ }^{28,29}$ as well as by STN stimulation. More recently, striatal dopamine cell transplantation in an animal model of PD also led to restoration of the neuronal firing and oscillations in the SN pars reticulata and STN to normal levels, confirming that continuous DA supply by VM grafts improves not only functional deficits but also rescues basal ganglia electrophysiological activity. ${ }^{30,31}$ Other experimental studies have shown that the characteristic increased subthalamic activity found in PD is coupled to abnormally elevated striatal levels of glutamate, which seems to be implicated in the pathogenesis and progression of the disease. ${ }^{32,33}$ Other authors have found that STN modulation leads to increased dopamine release in the SN and striatum, ${ }^{34}$ which is associated with the concomitant inhibition of pathological neuronal firing ${ }^{35}$ and a reduction in striatal glutamate levels. ${ }^{36}$ It has been proposed that by reducing the excitotoxic drive to the $\mathrm{SN}$, STN modulation could even halt disease progression. ${ }^{14,15}$ In the current work, STN-HFS boosted grafted cell survival by $180 \%$, fiber outgrowth by $140 \%$, and the overall graft volume by $230 \%$. This suggests that by reducing striatal neuronal firing and excitotoxicity, subthalamic neuromodulation provides a better environment for dopaminergic precursor cells, which, once transplanted, develop and integrate into the host striatum. ${ }^{11}$

\section{Neurotransplantation}

Despite its success in managing PD symptoms, it remains an open question whether STN stimulation affects the natural disease progression. ${ }^{37}$ As shown in studies with longterm postoperative follow-ups, patients gradually develop speech deficits, postural instability, akinesia, freezing, and gait disturbances, which are noticeable between 1 and 5 years after the procedure. ${ }^{38,39}$ Thus, cell-based therapy remains a potential clinical approach, particularly if alternative cell sources to embryonic tissue can be found. ${ }^{40}$ Cellbased therapy has shown proof-of-principle in animal models and open-label human studies, providing restoration of intrinsic dopamine delivery and stabilization or even improvement of motor symptoms following striatal transplantation. ${ }^{9,41}$ However, double-blind clinical trials have failed to show a consistent positive effect of grafting in comparison to sham-surgery. ${ }^{42,43}$ This paradox is probably explained by the use of different protocols, with distinct tissue dissection, management, and surgical techniques. ${ }^{10}$ Further development and standardization of tissue preparation into a single cell-suspension, as well as the advent of microtransplantation techniques, led to a marked increase in the cell viability, graft volume, and $\mathrm{TH}+$ fiber density of grafted cells. ${ }^{44}$ Nevertheless, the survival rate of transplanted dopaminergic cells remains below optimal expectations, which is an issue that needs addressing. In this sense, our study showed that STN neuromodulation provides a notable increment in the number of surviving transplanted cells and also has a positive impact on graft-host integration and functional recovery. In line with this, immunohistochemical assessment of graft-induced microglia activation in the host striatum revealed an increased number of ED-1+ cells within and surrounding the graft. Notwithstanding, in the DBS-TX group a significantly milder graft-versus-host reaction was observed, a fact that might also have an impact on the number of surviving transplanted cells. Other authors have shown that electric stimulation of deep brain structures, such as ventrolateral portion of hypothalamic ventromedial nuclei, subthalamic nucleus, and nucleus accumbens, induces alteration of systemic inflammatory response, changing circulatory levels of corticosterone and proinflammatory cytokines. ${ }^{45-48}$ However, data regarding microglial activation local changes following DBS are lacking in the current literature and would be worthy of further investigation in preclinical studies. Taken together, our results suggest that a reduced amount of dopaminergic cells, that is, embryonic tissue, would be necessary to restore dopamine production and relieve symptoms when transplantation is combined with STN-HFS beforehand.

\section{Behavioral Assessment}

In the unilateral MFB lesion model, rotational asymmetries are attributed to the dopamine imbalance between the ipsilateral and the contralateral striatum. ${ }^{19}$ In the case of the direct dopamine agonist apomorphine, activation of the hypersensitive receptors on the dopamine-depleted striatum leads to contralateral rotation. Although an effect was observed in both grafted groups 6 weeks following transplantation, STN-HFS led to faster recovery and significant improvement was observed as early as 2 weeks later. The 
amelioration in the postgraft sessions indicates that the dopamine released by the grafted cells was sufficient to influence the functional state of the DA postsynaptic receptors in the denervated striatum. ${ }^{49}$ The difference observed between groups at the second week suggests a positive combined STN-HFS + graft effect on the surviving dopaminergic grafted cells in the striatum, and on the rescue of dopamine-producing cells in the midbrain. ${ }^{14,15,50-52}$

Nevertheless, in the current study, STN neuromodulation improved limb-use asymmetry in the cylinder test even before transplantation. The assessment of forelimb akinesia has translational clinical relevance and may be analogous to limb akinesia and the difficulty of movement initiation often seen in patients with degeneration of nigrostriatal dopaminergic neurons. ${ }^{53,54}$ Compared to the transplantation-only group, the DBS-TX group achieved significantly better improvement in contralateral forelimb use at 2 and 6 weeks after transplantation. Most remarkably, the combined treatment resulted in control-level performance on the stepping test. To the best of our knowledge, normalization of behavior on this task has not been previously described in transplantation-only studies, which usually report partial or no effects on the stepping test, despite the fact that the transplanted cells spread over the whole striatum..$^{22,54-56}$

\section{Electric-Neurogenic Coupling and Future Perspectives}

Recently, excitation-neurogenesis coupling has been shown to foster differentiation of neuronal progenitors in hippocampal slices. ${ }^{13}$ Similarly, the positive effect of electrical activity on the maturation of neuronal progenitors has also been reported. ${ }^{57}$ Previous studies from our research group have shown that this neurogenic signaling depends on activation of voltage-dependent $\mathrm{Na}^{+}$-channels, but more so on a strong activation of L-type $\mathrm{Ca}^{2+}$-channels. ${ }^{58}$ This activation leads to CREB phosphorylation, which is also an important transcription factor involved in neurogenesis in vivo. ${ }^{59}$ In a 6-OHDA rat model of PD, STN-HFS was shown to induce changes on adult brain neurogenesis, increasing cell survival of newly formed cells in the olfactory bulb, in the dentate gyrus of the hippocampus, and rescue of dopamine in the striatum of lesioned rats. ${ }^{60}$ Our results showing that STN-HFS significantly increased cell proliferation index within the striatal graft and also in regions of constitutive neurogenesis, such as the RMS, are consistent with those reports. Taken together, there is robust evidence in the literature supporting a key role for electrical activity in the maturation of neuronal progenitors in vitro, but also in physiological neurogenesis. The question that arises from this evidence is whether electrical activity through chronic implants can also foster the differentiation and survival of fetal grafts in the host striatum. In fact, our data support this notion and open the intriguing and new possibility of associative therapies.
In line with this, although still frequently unrecognized, nonmotor symptoms (NMS) of PD have been associated to as much, if not more, morbidity as its classic motor features. ${ }^{61,62}$ Recently, DBS has been reported to improve quality of life and some of the NMS of the disease, however mainly indirectly, due to motor improvement or reduction of medicamentous therapy. ${ }^{38,63}$ On the other hand, preclinical works have indicated neuronal grafting to be capable of alleviating cognitive deficits or even restoring more complex cognitive function in neurodegenerative disorders; however, evidences supporting this concept are still sparse ${ }^{64-66}$ Therefore, the rationale of combining both therapeutic methods, that is, neuronal transplantation and electric neuromodulation, is worthy of further investigation and seems to be sustained by preclinical indications of their complementary effects, which could lead to relief of impairments in both motor and nonmotor domains of the disease.

\section{Conclusion}

In our experimental PD model, survival of dopaminergic grafted cells and functional recovery were observed after intrastriatal transplantation, and significantly improved when combined with STN stimulation. Although the precise mechanisms underlying these observations are not completely understood, the process seems to involve excitation-neurogenesis coupling and a decrease of striatal neuronal firing and excitotoxicity. Our data show that STN-HFS + transplantation can act synergistically; furthermore, the better graft survival and re-innervation with the host resulted in faster behavioral recovery in our model. The translation of preclinical studies to clinical application must always be considered with caution; however, our findings might open an unexplored road to combining neuromodulative and neuroregenerative strategies to treat severe neurologic conditions in the future.

\section{Acknowledgments}

We thank the technical support of Johanna Wessolleck and Marlene Löffler.

\section{Declaration of Conflicting Interests}

The author(s) declared no potential conflicts of interest with respect to the research, authorship, and/or publication of this article.

\section{Funding}

The author(s) disclosed receipt of the following financial support for the research, authorship, and/or publication of this article: This research was partly supported by the Stereotactic and Functional Neurosurgery Department, University Hospital, Freiburg, Germany, and the Brain-Links-Brain Tools Cluster of Excellence funded by the German Research Foundation (DFG, Grant Number EXC 1086). LLF has a grant from the Deutscher Akademischer 
Austauschdienst (DAAD) and Brazilian National Council for Scientific and Technological Development (CNPq).

\section{References}

1. Follett KA, Weaver FM, Stern M, et al. Pallidal versus subthalamic deep-brain stimulation for Parkinson's disease. $N$ Engl J Med. 2010;362:2077-2091.

2. Strauss I, Kalia SK, Lozano AM. Where are we with surgical therapies for Parkinson's disease? Parkinsonism Relat Disord. 2014;20(suppl 1):S187-S191.

3. Deuschl G, Schade-Brittinger C, Krack P, et al. A randomized trial of deep-brain stimulation for Parkinson's disease. $N$ Engl JMed. 2006;355:896-908.

4. Moro E, Lozano AM, Pollak P, et al. Long-term results of a multicenter study on subthalamic and pallidal stimulation in Parkinson's disease. Mov Disord. 2010;25:578-586.

5. Schuepbach WMM, Rau J, Knudsen K, et al. Neurostimulation for Parkinson's disease with early motor complications. $N$ Engl J Med. 2013;368:610-622.

6. McIntyre CC, Savasta M, Walter BL, Vitek JL. How does deep brain stimulation work? Present understanding and future questions. J Clin Neurophysiol. 2004;21:40-50.

7. Miocinovic S, Somayajula S, Chitnis S, Vitek JL. History, applications, and mechanisms of deep brain stimulation. JAMA Neurol. 2013;70:163-171.

8. Barker RA, Barrett J, Mason SL, Björklund A. Fetal dopaminergic transplantation trials and the future of neural grafting in Parkinson's disease. Lancet Neurol. 2013;12:84-91.

9. Kefalopoulou Z, Politis M, Piccini P, et al. Long-term clinical outcome of fetal cell transplantation for Parkinson disease: two case reports. JAMA Neurol. 2014;71:83-87.

10. Winkler C, Kirik D, Björklund A. Cell transplantation in Parkinson's disease: how can we make it work? Trends Neurosci. 2005;28:86-92.

11. Cordeiro KK, Cordeiro JG, Furlanetti LL, et al. Subthalamic nucleus lesion improves cell survival and functional recovery following dopaminergic cell transplantation in parkinsonian rats. Eur J Neurosci. 2014;39:1474-1484.

12. Ge S, Goh ELK, Sailor KA, Kitabatake Y, Ming G, Song H. GABA regulates synaptic integration of newly generated neurons in the adult brain. Nature. 2006;439:589-593.

13. Tozuka Y, Fukuda S, Namba T, Seki T, Hisatsune T. GABAergic excitation promotes neuronal differentiation in adult hippocampal progenitor cells. Neuron. 2005;47: 803-815.

14. Carvalho GA, Nikkhah G. Subthalamic nucleus lesions are neuroprotective against terminal 6-OHDA-induced striatal lesions and restore postural balancing reactions. Exp Neurol. 2001;171:405-417.

15. Spieles-Engemann AL, Behbehani MM, Collier TJ, et al. Stimulation of the rat subthalamic nucleus is neuroprotective following significant nigral dopamine neuron loss. Neurobiol Dis. 2010;39:105-115.

16. Lindemann C, Krauss JK, Schwabe K. Deep brain stimulation of the subthalamic nucleus in the 6-hydroxydopamine rat model of Parkinson's disease: effects on sensorimotor gating. Behav Brain Res. 2012;230:243-250.

17. Pruszak J, Just L, Isacson O, Nikkhah G. Isolation and culture of ventral mesencephalic precursor cells and dopaminergic neurons from rodent brains. Curr Protoc Stem Cell Biol. 2009; Chapter 2:Unit 2D.5.

18. Nikkhah G, Rosenthal C, Falkenstein G, Roedter A, Papazoglou A, Brandis A. Microtransplantation of dopaminergic cell suspensions: further characterization and optimization of grafting parameters. Cell Transplant. 2009;18:119-133.

19. Ungerstedt U. Striatal dopamine release after amphetamine or nerve degeneration revealed by rotational behaviour. Acta Physiol Scand Suppl. 1971;367:49-68.

20. García J, Carlsson T, Döbrössy M, Nikkhah G, Winkler C. Impact of dopamine to serotonin cell ratio in transplants on behavioral recovery and L-DOPA-induced dyskinesia. Neurobiol Dis. 2011;43:576-587.

21. Schallert T, Fleming SM, Leasure JL, Tillerson JL, Bland ST. CNS plasticity and assessment of forelimb sensorimotor outcome in unilateral rat models of stroke, cortical ablation, parkinsonism and spinal cord injury. Neuropharmacology. 2000;39:777-787.

22. Rödter A, Winkler C, Samii M, Nikkhah G. Complex sensorimotor behavioral changes after terminal striatal 6-OHDA lesion and transplantation of dopaminergic embryonic micrografts. Cell Transplant. 2000;9:197-214.

23. Schallert T, Norton D, Jones T. A clinically relevant unilateral rat model of Parkinsonian akinesia. J Neural Transplant Plast. 1992;3:323-333.

24. Benabid AL, Koudsié A, Pollak P, et al. Future prospects of brain stimulation. Neurol Res. 2000;22:237-246.

25. Benazzouz A, Hallett M. Mechanism of action of deep brain stimulation. Neurology. 2000;55:S13-S16.

26. Hirsch EC, Jenner P, Przedborski S. Pathogenesis of Parkinson's disease. Mov Disord. 2013;28:24-30.

27. De Solages C, Hill BC, Koop MM, Henderson JM, BronteStewart H. Bilateral symmetry and coherence of subthalamic nuclei beta band activity in Parkinson's disease. Exp Neurol. 2010;221:260-266.

28. López-Azcárate J, Tainta M, Rodríguez-Oroz MC, et al. Coupling between beta and high-frequency activity in the human subthalamic nucleus may be a pathophysiological mechanism in Parkinson's disease. J Neurosci. 2010;30: 6667-6677.

29. Priori A, Foffani G, Pesenti A, et al. Rhythm-specific pharmacological modulation of subthalamic activity in Parkinson's disease. Exp Neurol. 2004;189:369-379.

30. Rumpel R, Alam M, Klein A, et al. Neuronal firing activity and gene expression changes in the subthalamic nucleus after transplantation of dopamine neurons in hemiparkinsonian rats. Neurobiol Dis. 2013;59:230-243.

31. Gilmour TP, Piallat B, Lieu CA, et al. The effect of striatal dopaminergic grafts on the neuronal activity in the substantia nigra pars reticulata and subthalamic nucleus in hemiparkinsonian rats. Brain. 2011;134:3276-3289.

32. Hamani C, Saint-Cyr JA, Fraser J, Kaplitt M, Lozano AM. The subthalamic nucleus in the context of movement disorders. Brain. 2004;127:4-20.

33. Gubellini P, Eusebio A, Oueslati A, Melon C, Kerkerian-Le Goff L, Salin P. Chronic high-frequency stimulation of the subthalamic nucleus and L-DOPA treatment in experimental parkinsonism: effects on motor behaviour and striatal glutamate transmission. Eur J Neurosci. 2006;24: 1802-1814. 
34. Lee KH, Blaha CD, Harris BT, et al. Dopamine efflux in the rat striatum evoked by electrical stimulation of the subthalamic nucleus: potential mechanism of action in Parkinson's disease. Eur J Neurosci. 2006;23:1005-1014.

35. Benazzouz A, Piallat B, Pollak P, Benabid AL. Responses of substantia nigra pars reticulata and globus pallidus complex to high frequency stimulation of the subthalamic nucleus in rats: electrophysiological data. Neurosci Lett. 1995;189:77-80.

36. Walker RH, Koch RJ, Sweeney JE, Moore C, Meshul CK. Effects of subthalamic nucleus lesions and stimulation upon glutamate levels in the dopamine-depleted rat striatum. Neuroreport. 2009;20:770-775.

37. Deuschl G, Paschen S, Witt K. Clinical outcome of deep brain stimulation for Parkinson's disease. Handb Clin Neurol. 2013;116:107-128.

38. Fasano A, Daniele A, Albanese A. Treatment of motor and non-motor features of Parkinson's disease with deep brain stimulation. Lancet Neurol. 2012;11:429-442.

39. Rizzone MG, Fasano A, Daniele A, et al. Long-term outcome of subthalamic nucleus DBS in Parkinson's disease: from the advanced phase towards the late stage of the disease? Parkinsonism Relat Disord. 2014;20:376-381.

40. Dunnett SB, Rosser AE. Challenges for taking primary and stem cells into clinical neurotransplantation trials for neurodegenerative disease. Neurobiol Dis. 2014;61:79-89.

41. Döbrössy M, Busse M, Piroth T, Rosser A, Dunnett S, Nikkhah G. Neurorehabilitation with neural transplantation. Neurorehabil Neural Repair. 2010;24:692-701.

42. Freed CR, Greene PE, Breeze RE, et al. Transplantation of embryonic dopamine neurons for severe Parkinson's disease. N Engl J Med. 2001;344:710-719.

43. Olanow CW, Goetz CG, Kordower JH, et al. A double-blind controlled trial of bilateral fetal nigral transplantation in Parkinson's disease. Ann Neurol. 2003;54:403-414.

44. Nikkhah G, Olsson M, Eberhard J, Bentlage C, Cunningham MG, Björklund A. A microtransplantation approach for cell suspension grafting in the rat Parkinson model: a detailed account of the methodology. Neuroscience. 1994;63: 57-72.

45. Calleja-Castillo JM, De La Cruz-Aguilera DL, Manjarrez J, et al. Chronic deep brain stimulation of the hypothalamic nucleus in Wistar rats alters circulatory levels of corticosterone and proinflammatory cytokines. Clin Dev Immunol. 2013;2013:698634.

46. Seifried C, Boehncke S, Heinzmann J, et al. Diurnal variation of hypothalamic function and chronic subthalamic nucleus stimulation in Parkinson's disease. Neuroendocrinology. 2013;97:283-290.

47. Novakova L, Haluzik M, Jech R, Urgosik D, Ruzicka F, Ruzicka E. Hormonal regulators of food intake and weight gain in Parkinson's disease after subthalamic nucleus stimulation. Neuro Endocrinol Lett. 2011;32:437-441.

48. De Koning PP, Figee M, Endert E, Storosum JG, Fliers E, Denys D. Deep brain stimulation for obsessive-compulsive disorder is associated with cortisol changes. Psychoneuroendocrinology. 2013;38:1455-1459.

49. Abrous DN, Shaltot AR, Torres EM, Dunnett SB. Dopaminerich grafts in the neostriatum and/or nucleus accumbens: effects on drug-induced behaviours and skilled paw-reaching. Neuroscience. 1993;53:187-197.
50. He Z, Jiang $\mathrm{Y}, \mathrm{Xu} \mathrm{H}$, et al. High frequency stimulation of subthalamic nucleus results in behavioral recovery by increasing striatal dopamine release in 6-hydroxydopamine lesioned rat. Behav Brain Res. 2014;263:108-114.

51. Lee KH, Kristic K, van Hoff R, et al. High-frequency stimulation of the subthalamic nucleus increases glutamate in the subthalamic nucleus of rats as demonstrated by in vivo enzymelinked glutamate sensor. Brain Res. 2007;1162:121-129.

52. Meissner W, Harnack D, Reese R, et al. High-frequency stimulation of the subthalamic nucleus enhances striatal dopamine release and metabolism in rats. J Neurochem. 2003;85:601-609.

53. Dunnett SB, Robbins TW. The functional role of mesotelencephalic dopamine systems. Biol Rev Camb Philos Soc. 1992;67:491-518.

54. Olsson M, Nikkhah G, Bentlage C, Björklund A. Forelimb akinesia in the rat Parkinson model: differential effects of dopamine agonists and nigral transplants as assessed by a new stepping test. J Neurosci. 1995;15:3863-3875.

55. Kirik D, Winkler C, Björklund A. Growth and functional efficacy of intrastriatal nigral transplants depend on the extent of nigrostriatal degeneration. J Neurosci. 2001;21:2889-2896.

56. Winkler C, Bentlage C, Nikkhah G, Samii M, Björklund A. Intranigral transplants of GABA-rich striatal tissue induce behavioral recovery in the rat Parkinson model and promote the effects obtained by intrastriatal dopaminergic transplants. Exp Neurol. 1999;155:165-186.

57. Deisseroth K, Singla S, Toda H, Monje M, Palmer TD, Malenka RC. Excitation-neurogenesis coupling in adult neural stem/progenitor cells. Neuron. 2004;42:535-552.

58. Lepski G, Jannes CE, Nikkhah G, Bischofberger J. cAMP promotes the differentiation of neural progenitor cells in vitro via modulation of voltage-gated calcium channels. Front Cell Neurosci. 2013;7:155.

59. Giachino C, De Marchis S, Giampietro C, et al. cAMP response element-binding protein regulates differentiation and survival of newborn neurons in the olfactory bulb. $J$ Neurosci. 2005;25:10105-10118.

60. Khaindrava V, Salin P, Melon C, Ugrumov M, Kerkerian-LeGoff L, Daszuta A. High frequency stimulation of the subthalamic nucleus impacts adult neurogenesis in a rat model of Parkinson's disease. Neurobiol Dis. 2011;42:284-291.

61. Wolters E, Baumann C. Parkinson Disease and Other Movement Disorders: Motor Behavioural Disorders and Behavioural Motor Disorders. Amsterdam, Netherlands: VU University Press; 2013.

62. Chaudhuri KR, Odin P, Antonini A, Martinez-Martin P. Parkinson's disease: the non-motor issues. Parkinsonism Relat Disord. 2011;17:717-723.

63. Klingelhoefer L, Samuel M, Chaudhuri KR, Ashkan K. An update of the impact of deep brain stimulation on non motor symptoms in Parkinson's disease. J Parkinsons Dis. 2014;4:289-300.

64. Isacson O, Dunnett SB, Björklund A. Graft-induced behavioral recovery in an animal model of Huntington disease. Proc Natl Acad Sci U S A. 1986;83:2728-2732.

65. Dunnett SB, White A. Striatal grafts alleviate bilateral striatal lesion deficits in operant delayed alternation in the rat. Exp Neurol. 2006;199:479-489.

66. Lelos MJ, Dowd E, Dunnett SB. Nigral grafts in animal models of Parkinson's disease. Is recovery beyond motor function possible? Prog Brain Res. 2012;200:113-142. 